\title{
Cellular Reprogramming toward the Erythroid Lineage
}

\author{
Laura J. Norton, Alister P. W. Funnell, Richard C. M. Pearson, and Merlin Crossley \\ School of Biotechnology and Biomolecular Sciences, University of New South Wales, Sydney, NSW 2052, Australia \\ Correspondence should be addressed to Merlin Crossley, m.crossley@unsw.edu.au
}

Received 30 March 2011; Accepted 8 May 2011

Academic Editor: Michael Föller

Copyright ( 2011 Laura J. Norton et al. This is an open access article distributed under the Creative Commons Attribution License, which permits unrestricted use, distribution, and reproduction in any medium, provided the original work is properly cited.

\begin{abstract}
Haemoglobinopathies such as thalassaemia and sickle cell disease present a major health burden. Currently, the main forms of treatment for these diseases are packed red blood cell transfusions and the administration of drugs which act to nonspecifically reactivate the production of foetal haemoglobin. These treatments are ongoing throughout the life of the patient and are associated with a number of risks, such as limitations in available blood for transfusion, infections, iron overload, immune rejection, and side effects associated with the drug treatments. The field of cellular reprogramming has advanced significantly in the last few years and has recently culminated in the successful production of erythrocytes in culture. This paper will discuss cellular reprogramming and its potential relevance to the treatment of haemoglobinopathies.
\end{abstract}

\section{Introduction: Globin Gene Regulation and Haemoglobinopathies}

The various blood cell lineages in mammals arise from a multipotent haematopoietic stem cell via particular differentiation pathways. One of these pathways, erythropoiesis, leads to the production of red blood cells (RBCs), which transport oxygen and carbon dioxide around the body by means of the intracellular metalloprotein haemoglobin $(\mathrm{Hb})$. $\mathrm{Hb}$ is a tetramer, consisting of two $\alpha$-globin and two $\beta$-globin subunits. In mammals, these globin chains are encoded by two gene loci: the $\alpha$-globin locus and the $\beta$-globin locus. In humans, the $\alpha$-globin locus consists of the embryonic $\zeta$ - and adult $\alpha$-globin genes, and the $\beta$-globin locus comprises the embryonic $\varepsilon_{-}^{-}$, foetal $\gamma^{G_{-}}$and $\gamma^{A}$, and adult $\delta$ - and $\beta$-globin genes $[1,2]$. The globin genes expressed from these loci differ from embryonic to adult erythropoiesis in order to meet varying oxygen demands and facilitate placental transfer of oxygen from mother to embryo [3].

There are a number of severe diseases caused by the disruption of adult globin genes, including thalassaemias and certain types of anaemia. According to the World Health Organisation, approximately $5 \%$ of the world's population carry genes involved in $\mathrm{Hb}$ disorders, and as such, they present an enormous health burden. Thalassaemia is caused by a reduction or abolition of the expression of one or more globin genes, resulting in an imbalance of $\alpha$ - and $\beta$-globin chains in red blood cells and consequent anaemia $[1,4]$. Sickle cell anaemia is another prevalent haemoglobinopathy and is caused by a mutation in the adult $\beta$-globin gene which generates a single glutamic acid to valine amino acid substitution. This mutation leads to the polymerisation of globins in venous circulation $[5,6]$, which can trigger a rigid and sickled cell phenotype $[7,8]$ and results in a number of acute conditions such as vaso-occlusion, splenic sequestration, and haemolytic anaemia [9].

There are currently a number of treatments available for patients suffering from thalassaemia and sickle cell anaemia. The most common is packed red blood cell transfusion, but this is associated with a number of problems, such as sufficiency of supply, bacterial and viral infection, biochemical and biomechanical changes during storage (red blood cell storage lesions), and the risk of immune rejection from the patient $[10,11]$. Furthermore, blood transfusions are ongoing throughout a patient's life and often lead to a potentially fatal buildup of iron and associated reduction in organ activity.

Another potential therapeutic option involves the reactivation of foetal $\gamma$-globin expression in adult patients. Residual production of foetal $\gamma$-globin persists naturally 
throughout life, and levels vary between individuals $[12,13]$. This persistent expression allows two $\gamma$-globin chains to combine with two adult $\alpha$-globin chains to form what is known as foetal $\mathrm{Hb}(\mathrm{HbF})$. As only the adult $\beta$-globin gene is mutated in sickle cell anaemia, affected infants are protected from severe symptoms until they reach several months of age, due to the large amount of $\mathrm{HbF}$ still in circulation at birth [14]. Furthermore, patients who have inherited alleles associated with increased levels of $\mathrm{HbF}$, known as hereditary persistence of foetal $\mathrm{Hb}(\mathrm{HPFH})$, are protected into adulthood [15]. Similarly, a more asymptomatic disease phenotype has also been shown in patients with $\beta$-thalassaemia who exhibit higher levels of $\mathrm{HbF}$ [16]. Together, these observations indicate that increased foetal $\gamma$-globin is able to compensate in part for the loss of adult $\beta$-globin function and thus ameliorates the symptoms of certain adult haemoglobinopathies. Accordingly, a number of drug treatments for $\beta$-thalassaemia and sickle cell disease, for instance, 5-azacitidine, hydroxyurea, and butyrate, all act by nonspecifically reactivating foetal $\gamma$-globin gene expression by various mechanisms. The effects of these drug treatments are transient and thus require ongoing administration. There is evidence that longterm administration of these drugs has chronic side effects, consistent with their lack of specificity $[8,17]$.

As the existing methods of treatment for these haemoglobinopathies remain inadequate, alternative forms of therapy are currently being sought, and stem cell therapies should be considered. This paper will discuss progress in utilising novel cellular reprogramming techniques to treat $\mathrm{RBC}$ diseases.

\section{Cellular Reprogramming}

Stem cells, both embryonic and adult, have the ability to differentiate into various cell types, making them a potentially attractive treatment option. Embryonic stem cells (ESCs) and adult stem cells (ASCs) each have their own strengths and disadvantages in these strategies. ESCs are more easily grown in culture and are pluripotent, meaning that they are able to differentiate into any cell of the body. The practicality of widespread ESC use for therapeutic purposes, however, has been questioned due to issues of supply and ethical and legal considerations. Moreover, these cells carry the risk of allogeneic immune rejection. ASCs, on the other hand, overcome some of these problems as they can be harvested from each individual patient. These cells, however, offer a different set of challenges. They are not abundant and are difficult to obtain, often being harboured in internal organs such as the gut and bone marrow. They have proven difficult to culture in vitro [18], and furthermore, they are widely believed to be multipotent rather than pluripotent and are thus only able to differentiate into certain cell types [19]. Cellular reprogramming potentially overcomes these issues and may offer new treatment methods for a range of diseases, including those of RBCs.

2.1. Classic Cellular Reprogramming. Takahashi and Yamanaka [20] were the first to show that it is possible to take differentiated somatic cells and transform them into cells with pluripotent potential. They began by identifying a pool of 24 transcription factors which are important in maintaining stem cell traits and used retroviral transduction to express these factors in murine embryonic and adult fibroblasts. They found that these cells then displayed characteristics and properties comparable to those of pluripotent ESCs. They were able to further refine the required transcription factors by setting up numerous combinations to determine which were essential to this process and identified four factors, Oct4, Sox2, Klf4, and c-Myc, needed to transform a somatic cell to an induced pluripotent stem cell (iPSC). Takahashi et al. [21] then applied these four factors to human cells and transformed neonatal and adult human fibroblasts into human iPSCs (hiPSCs). This was a valuable subsequent step as it showed that this process can be reproduced with human cells and could thus potentially be utilised for stem cellbased therapies of human disease. Several other transcription factor combinations have since been shown to be sufficient to reprogram somatic cells [22-24] and one particular combination, involving OCT4, SOX2, NANOG, and LIN28, is particularly efficient and is now widely utilised [25]. Figure 1(a) depicts the process of cellular reprogramming.

There are several potential issues associated with using iPSCs to treat disease. For example, a number of the transcription factors used to generate these cells, including cMyc, Klf4, and Lin28, are oncogenes, and their misexpression can lead to cancer [26-28]. In order for iPSCs to be differentiated into a cell type of choice, the retrovirally transmitted genes need to be switched off or removed to reduce the possibility of their inducing tumours [22, 29]. Another potential issue is that the transplantation of any cells that have not been fully differentiated from the iPSC state could lead to the formation of cancerous teratomas. Any cells remaining which are still in a pluripotent state could multiply and, without the appropriate growth controls, would have the potential to result in tumours in transplant patients. To potentially avoid the issue of tumour formation and uncontrolled proliferation of iPSCs, an alternative methodology known as transdifferentiation is also being considered as a potential treatment strategy.

2.2. Transdifferentiation. Transdifferentiation is achieved by introducing various exogenous factors into a differentiated cell, such as a fibroblast, to directly convert it into another type of differentiated cell, thereby bypassing the pluripotent state (Figure 1(b)). As early as 1990, Choi et al. [30] were able to convert various cells types, including dermal fibroblasts and chondroblasts, into mononucleated, striated myoblasts that were indistinguishable from normal myoblasts in vivo. This was achieved through the expression of the myogenic regulatory factor $\mathrm{MyoD}$, a transcription factor known to be involved in the determination of muscle cells. Shortly after this, another group showed that it was possible to turn myeloid 416B cells into mast cells through the forced expression of GATA-2 and GATA-3, two transcription factors which play important roles in haematopoiesis [31]. Halder et al. [32] investigated the effects of ectopically expressing the gene eyeless (ey), an orthologue of the mammalian Pax6 gene, 


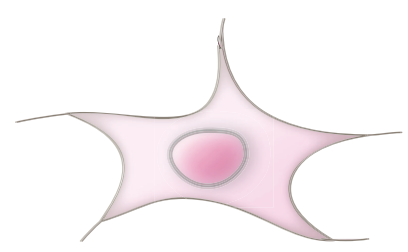

Fibroblast

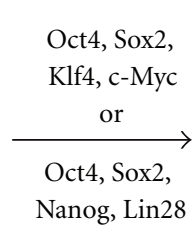

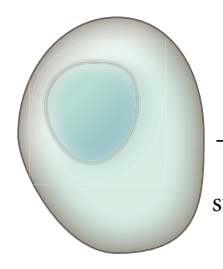

iPSC

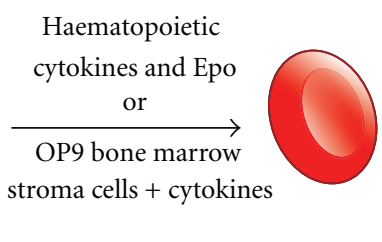

Erythrocyte

(a) Classic cellular reprogramming

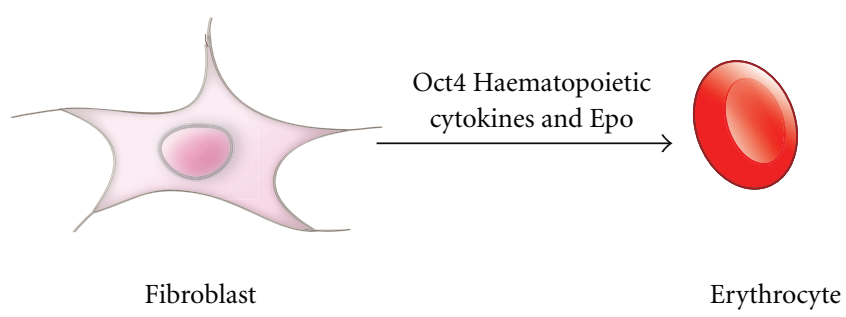

(b) Transdifferentiation

FIGURE 1: Diagrammatic representation of cellular reprogramming compared to transdifferentiation in the development of erythrocytes. (a) Fibroblasts cells can be reprogrammed into pluripotent cells through the introduction of various exogenous factors. From this induced pluripotent state, these cells can be either cultured with various cytokines supporting erythrocyte differentiation and growth, for instance, IL-3, IL-6, and Erythropoietin (Epo) or cultured with OP9 bone marrow stroma cells to differentiate into erythrocytes. (b) Fibroblasts cells can be reprogrammed directly into erythrocytes through the introduction of Oct4 and culturing with Epo in conjunction with other haematopoietic cytokines.

in Drosophila and found that eye structures formed in places such as the wings and legs.

Since Takahashi and Yamanaka's pioneering studies in cellular reprogramming, the field of transdifferentiation has advanced considerably. Zhou et al. [33] investigated the effects of expressing Ngn3, Pdx1, and Mafa, transcription factors involved in $\beta$-cell differentiation, on exocrine cells of the adult pancreas. They found that the coexpression of these factors was able to convert the exocrine cells into $\beta$-cells. The induced $\beta$-cells were identical to endogenous $\beta$-cells in morphology and showed similar expression of genes associated with $\beta$-cell function. These cells can also rescue the phenotype of hyperglycaemia, as they are able to secrete insulin and remodel surrounding vasculature. In other work, Vierbuchen et al. [34] utilised the neuralspecific transcription factors, Ascl1, Brn2, and Myt11, to rapidly convert both murine embryonic fibroblasts and adult fibroblasts directly into functional neurons. These induced neuronal cells express neuron-specific proteins, generate action potentials, and form functional synapses. A further advance has shown that it is also possible to transdifferentiate fibroblasts into functional neural progenitor cells by transient induction of Oct4, Sox 2, Klf4, and c-Myc in cells cultured in a defined neural reprogramming medium [35]. This process bypassed the generation of iPSCs and gave rise to multipotent progenitors with the capacity to expand and differentiate into a number of neural lineages. All of these experiments indicate that it is possible to direct a differentiated cell to another cell fate through the application of extragenic factors.

\section{Cellular Reprogramming as a Potential Treatment for Anaemia}

Advances in cellular reprogramming have raised the attractive possibility that this technology could be utilised to generate a limitless source of immune-matched, pathogenfree erythrocytes for transfusion. Efforts were thus made to produce mature erythroid cells from hiPSCs in culture. An initial study by Feng et al. [28] revealed some practical difficulties. They found that hiPSCs are capable of generating haematopoietic cells with phenotypic and morphological characteristics similar to those derived from hESCs; however, these hiPSC-derived cells exhibited a dramatically reduced capacity (by greater than 1000-fold) to generate erythroid cells.

A subsequent study by Lapillone et al., however, showed that it was indeed possible to produce significant numbers of mature erythroid cells from hiPSCs in vitro [36]. This group employed the methods outlined by Thomson's group [25] using OCT4, SOX2, NANOG, and LIN28 to convert fibroblasts to hiPSCs. They then cultured these cells in medium containing the cytokines SCF, TPO, FLT3 ligand, rhu BMP4, rhu VEGF-A165, IL-3, IL-6, and erythropoietin (Epo). These culture conditions were optimised to obtain embryoid bodies that display early erythroid commitment. They analysed the expression profiles of these cells over 20 days of culture and found that pluripotent stem cell markers decreased whilst the erythroid markers CD36, CD235a, and CD71 increased. Cells at day 20 were found to have a high erythroid potential and were plated in sequential 
cocktails of cytokines comprising SCF, IL-3, and/or Epo. Erythroid maturation was achieved after another 25 days and was confirmed by morphological examination and by flow cytometric analysis of erythroid markers $\left(\mathrm{CD} 235 \mathrm{a}^{\text {hi }}, \mathrm{CD} 71^{\text {hi }}\right.$, and $\left.\mathrm{CD} 36^{\text {lo }}\right)$. Furthermore, these erythroid cells were able to enucleate, albeit with reduced capacity compared to hESCderived erythroid cells, and were found to express functional $\mathrm{Hb}$ (predominantly $\mathrm{HbF}$ ). These hiPSC-derived erythroid cells were compared to those differentiated from hESCs, and no significant differences were detected in terms of erythroid commitment, expression of erythroid markers, and type and functionality of $\mathrm{Hb}$.

The study by Lapillone et al. revealed that while functional RBCs could be generated from hiPSCs, when compared to hESCs, hiPSCs were shown to have reduced (approximately 8 -fold) amplification potential in producing mature erythroid cells. A recent study has set out to determine whether this reduced efficiency is an intrinsic property of hiPSCs, or whether it can be increased by altered culture conditions [37]. This study showed not only that it is possible to generate RBCs from hiPSCs with similar efficiency to hESCs, but also that these cells can be differentiated from hiPSCs generated using episomal vectors, obviating the need for potentially deleterious retroviral transfection. This group cultured hiPSCs derived from human fibroblasts with the OP9 bone marrow stroma line to induce hematopoietic differentiation and followed this with selective expansion of erythroid cells in serum-free media with cytokines supporting $\mathrm{RBC}$ differentiation. The erythroid cultures established in this study consisted of a pure population of CD235a+ CD45- leukocyte-free RBCs which had robust expansion capabilities, as well as a long lifespan of up to 90 days. These hiPSC-derived cells can enucleate and were shown to express foetal $\gamma$ - and embryonic $\mathcal{\varepsilon}$-globin demonstrating successful reprogramming of the $\beta$-globin locus. The results from this study show that it is possible to produce significant numbers of erythroid cells from fibroblast-derived hiPSCs, and that thus cellular reprogramming could contribute to the treatment of haemoglobinopathies. Furthermore, since these RBCs are generated from transgene-free hiPSCs, they circumvent problems associated with genomic integration and undesired reactivation of reprogramming factors.

Another recent study has shown that it is possible to use transdifferentiation to produce haematopoietic cell lineages, including mature erythrocytes [38]. In this work, Szabo et al. transduced human dermal fibroblasts with OCT4 alone and showed that these cells then express the panhaematopoietic marker CD45 but lack the pluripotency marker Tra-1-60. They then cultured these cells in a haematopoietic cytokine cocktail containing SCF, G-CSF, FLT3LG, IL-3, IL-6, and BMP-4 supplemented with Epo and observed an emergence of cells expressing adult $\beta$-globin protein and the red blood cell marker Glycophorin-A. Moreover, these erythroid cells were able to mature in culture and underwent enucleation. In addition, the authors noted that these erythroid cells express adult rather than embryonic globins, unlike cells derived from human pluripotent stem cells $[39,40]$, perhaps suggesting that they have indeed been transdifferentiated from adult fibroblasts, bypassing the hiPSC stage. As the techniques used in this study avoid the pluripotent state as well as have a high yield, expansion capacity, and clinical feasibility, this strategy could provide a reasonable basis for autologous cell replacement therapies.

These studies demonstrate that it is possible to differentiate hiPSCs into mature erythrocytes in vitro for transfusions. In order to utilise these methods to treat RBC diseases successfully, fibroblasts could potentially be taken from either a healthy immunomatched individual, or a universal donor, and be converted to erythrocytes via the hiPSC state. These cells could then be mass produced in culture and stored for transfusion. A study has already developed hiPSCs from an individual with a Bombay phenotype of the $\mathrm{ABO}$ blood group system, where the $\mathrm{ABH}$ antigen is not expressed on RBCs, and blood can be donated to anyone [41]. This group has further demonstrated that it is possible to differentiate haematopoietic lineages from these cells although they have not explicitly shown mature erythrocyte differentiation. This possible treatment method circumvents many of the problems associated with current transfusions, such as questionable quality, lack of supply, and immunerejection. However, issues still remain, such as cost and the risk of iron overloading.

\section{Cellular Reprogramming as a Potential Cure for Anaemia}

In order to cure, rather than treat, RBC diseases, healthy progenitor cells must be transplanted into patients and subsequently repopulate the haematopoietic system. A promising study has already shown that it is possible to use reprogrammed cells to treat sickle cell anaemia in mice. Hanna et al. [42] took cells from the tail tips of a humanized sickle cell anaemic mouse model, in which the mouse $\alpha$ globin genes have been replaced with human $\alpha$-globin, and the mouse $\beta$-globin genes have been replaced with human $\gamma^{A}$ and $\beta^{S}$ (sickle) globin genes [43], and through adaptation of the protocol developed by Takahashi and Yamanaka, who reprogrammed these cells into iPSCs. Once this was accomplished, they were able to correct the mutant $\beta^{S}$-globin gene by replacing it with a copy of the wildtype human $\beta$ globin gene through homologous recombination. Following this, the iPSCs were differentiated into haematopoietic progenitors through the ectopic expression of HoxB4, and by growing in haematopoietic cytokines on an OP9 bone marrow stroma cell line. These corrected haematopoietic progenitor cells were transplanted into the sickle mice after irradiation. This group then carried out extensive testing to determine whether the treated mice displayed any further signs of the disease. Firstly, functional correction was evaluated by electrophoresis for the human $\beta$-globin proteins $\gamma^{A}$ and $\beta^{S}$, and they found a significant increase in $\gamma^{A}$ - and a decrease in $\beta^{S}$-globin. Blood counts were also performed 12 weeks after transplant, which showed that treated mice had increased RBCs, $\mathrm{Hb}$, and packed cell volume as well as reduced reticulocytes, a common indicator of sickle cell disease and severity. Lastly, they examined symptomatic indicators of sickle cell anaemia such as urine concentration, body weight, and breathing rate and found that all three 
of these parameters were ameliorated in the treated mice. This study thus provides an important proof of principle that cellular reprogramming can be employed to correct erythroid disorders, albeit in this case, in conjunction with gene therapy.

\section{Conclusions}

Normal erythropoiesis is dependent upon the correct expression of globin genes. Where globin genes are incorrectly expressed, or are mutated, anaemia or thalassemia results. Current therapy for these disorders involves packed red blood cell transfusions, which are limited by supply, risk of infection, expense, and patient rejection. Drug-based therapies involve the nonspecific reactivation of foetal globins and have long-term side effects. In seeking alternative strategies, recent advances have shown that cellular reprogramming can now generate large quantities of red blood cells in culture, potentially for use in transfusions. Furthermore, these strategies have been successfully combined with gene therapy to treat a sickle cell anaemia mouse model, suggesting that cellular reprogramming will provide a realistic future alternative to conventional treatment of haemoglobinopathies.

\section{References}

[1] A. C. Perkins, K. R. Peterson, G. Stamatoyannopoulos, H. E. Witkowska, and S. H. Orkin, "Fetal expression of a human A $\gamma$ globin transgene rescues globin chain imbalance but not hemolysis in EKLF null mouse embryos," Blood, vol. 95, no. 5, pp. 1827-1833, 2000.

[2] T. Trimborn, J. Gribnau, F. Grosveld, and P. Fraser, "Mechanisms of developmental control transcription in the murine $\alpha$ and $\beta$-globin loci," Genes and Development, vol. 13, no. 1, pp. 112-124, 1999.

[3] P. A. Oneal, N. M. Gantt, J. D. Schwartz et al., "Fetal hemoglobin silencing in humans," Blood, vol. 108, no. 6, pp. 20812086, 2006.

[4] M. H. Baron, "Transcriptional control of globin gene switching during vertebrate development," Biochimica et Biophysica Acta-Gene Structure and Expression, vol. 1351, no. 1-2, pp. 51-72, 1997.

[5] O. Galkin and P. G. Vekilov, "Mechanisms of homogeneous nucleation of polymers of sickle cell anemia hemoglobin in deoxy state," Journal of Molecular Biology, vol. 336, no. 1, pp. 43-59, 2004.

[6] L. Pauling, H. A. Itano, S. J. Singer, and I. C. Wells, "Sickle cell anemia, a molecular disease," Science, vol. 110, no. 2865, pp. 543-548, 1949.

[7] P. S. Frenette and G. F. Atweh, "Sickle cell disease: old discoveries, new concepts, and future promise," Journal of Clinical Investigation, vol. 117, no. 4, pp. 850-858, 2007.

[8] R. Mabaera, R. J. West, S. J. Conine et al., "A cell stress signaling model of fetal hemoglobin induction: what doesn't kill red blood cells may make them stronger," Experimental Hematology, vol. 36, no. 9, pp. 1057-1072, 2008.

[9] O. S. Platt, D. J. Brambilla, W. F. Rosse et al., "Mortality in sickle cell disease. Life expectancy and risk factors for early death," New England Journal of Medicine, vol. 330, no. 23, pp. 1639-1644, 1994.

[10] J. Mountford, E. Olivier, and M. Turner, "Prospects for the manufacture of red cells for transfusion," British Journal of Haematology, vol. 149, no. 1, pp. 22-34, 2010.
[11] M. H. Antonelou, A. G. Kriebardis, and I. S. Papassideri, "Aging and death signalling in mature red cells: from basic science to transfusion practice," Blood Transfusion, vol. 8, supplement 3, pp. s39-s47, 2010.

[12] C. Garner, T. Tatu, J. E. Reittie et al., "Genetic influences on F cells and other hematologic variables: a twin heritability study," Blood, vol. 95, no. 1, pp. 342-346, 2000.

[13] S. L. Thein and S. Menzel, "Discovering the genetics underlying foetal haemoglobin production in adults," British Journal of Haematology, vol. 145, no. 4, pp. 455-467, 2009.

[14] J. Watson, "Sickling in Negro newborns-its possible relationship to fetal hemoglobin," American Journal of Medicine, vol. 5, no. 1, pp. 159-160, 1948.

[15] S. Friedman and E. Schwartz, "Hereditary persistence of fetal hemoglobin with beta-chain synthesis in cis position (ggamma-beta+-hpfh) in a Negro family," Nature, vol. 259, no. 5539, pp. 138-140, 1976

[16] D. J. Weatherall and J. B. Clegg, "Inherited haemoglobin disorders: an increasing global health problem," Bulletin of the World Health Organization, vol. 79, no. 8, pp. 704-712, 2001.

[17] R. M. Böhmer, "Reactivation of fetal hemoglobin in adult stem cell erythropoiesis by transforming growth factor- $\beta$," Journal of Hematotherapy and Stem Cell Research, vol. 12, no. 5, pp. 499-504, 2003.

[18] J. Czyz, C. Wiese, A. Rolletschek, P. Blyszczuk, M. Cross, and A. M. Wobus, "Potential of embryonic and adult stem cells in vitro," Journal of Biological Chemistry, vol. 384, no. 10-11, pp. 1391-1409, 2003.

[19] O. J. Borge, "Embryonic and adult stem cells," Acta Veterinaria Scandinavica, vol. 99, pp. 39-43, 2004.

[20] K. Takahashi and S. Yamanaka, "Induction of pluripotent stem cells from mouse embryonic and adult fibroblast cultures by defined factors," Cell, vol. 126, no. 4, pp. 663-676, 2006.

[21] K. Takahashi, K. Tanabe, M. Ohnuki et al., "Induction of pluripotent stem cells from adult human fibroblasts by defined factors," Cell, vol. 131, no. 5, pp. 861-872, 2007.

[22] S. Yamanaka, M. Nakagawa, M. Koyanagi et al., "Generation of induced pluripotent stem cells without Myc from mouse and human fibroblasts," Nature Biotechnology, vol. 26, no. 1, pp. 101-106, 2008.

[23] H. H. Ng, J. C. D. Heng, B. Feng et al., "The Nuclear receptor $\mathrm{Nr} 5 \mathrm{a} 2$ can replace Oct 4 in the reprogramming of murine somatic cells to pluripotent cells," Cell Stem Cell, vol. 6, no. 2, pp. 167-174, 2010.

[24] K. Plath, R. Ho, and C. Chronis, "Mechanistic insights into reprogramming to induced pluripotency," Journal of Cellular Physiology, vol. 226, no. 4, pp. 868-878, 2011.

[25] J. Yu, M. A. Vodyanik, K. Smuga-Otto et al., "Induced pluripotent stem cell lines derived from human somatic cells," Science, vol. 318, no. 5858, pp. 1917-1920, 2007.

[26] J. A. West, S. R. Viswanathan, A. Yabuuchi et al., "A role for Lin28 in primordial germ-cell development and germ-cell malignancy," Nature, vol. 460, article U151, no. 7257, pp. 909913, 2009.

[27] S. R. Viswanathan, J. T. Powers, W. Einhorn et al., "Lin28 promotes transformation and is associated with advanced human malignancies," Nature Genetics, vol. 41, article U109, no. 7, pp. 843-848, 2009.

[28] Q. Feng, S. J. Lu, I. Klimanskaya et al., "Hemangioblastic derivatives from human induced pluripotent stem cells exhibit limited expansion and early senescence," Stem Cells, vol. 28, no. 4, pp. 704-712, 2010.

[29] K. Okita, T. Ichisaka, and S. Yamanaka, "Generation of germline-competent induced pluripotent stem cells," Nature, vol. 448, no. 7151, pp. 313-317, 2007. 
[30] J. Choi, M. L. Costa, C. S. Mermelstein, C. Chagas, S. Holtzer, and H. Holtzer, "MyoD converts primary dermal fibroblasts, chondroblasts, smooth muscle, and retinal pigmented epithelial cells into striated mononucleated myoblasts and multinucleated myotubes," Proceedings of the National Academy of Sciences of the United States of America, vol. 87, no. 20, pp. 7988-7992, 1990.

[31] J. Visvader and J. M. Adams, "Megakaryocytic differentiation induced in 416B myeloid cells by GATA-2 and GATA-3 transgenes or 5-azacytidine is tightly coupled to GATA-1 expression," Blood, vol. 82, no. 5, pp. 1493-1501, 1993.

[32] G. Halder, P. Callaerts, and W. J. Gehring, "Induction of ectopic eyes by targeted expression of the eyeless gene in Drosophila," Science, vol. 267, no. 5205, pp. 1788-1792, 1995.

[33] Q. Zhou, J. Brown, A. Kanarek, J. Rajagopal, and D. A. Melton, "In vivo reprogramming of adult pancreatic exocrine cells to [bgr]-cells," Nature, vol. 455, no. 7213, pp. 627-632, 2008.

[34] T. Vierbuchen, A. Ostermeier, Z. P. Pang, Y. Kokubu, T. C. Südhof, and M. Wernig, "Direct conversion of fibroblasts to functional neurons by defined factors," Nature, vol. 463, no. 7284, pp. 1035-1041, 2010.

[35] J. Kim, J. A. Efe, S. Zhu et al., "Direct reprogramming of mouse fibroblasts to neural progenitors," Proceedings of the National Academy of Sciences of the United States of America, vol. 108, no. 19, pp. 7838-7843, 2011.

[36] H. Lapillonne, L. Kobari, C. Mazurier et al., "Red blood cell generation from human induced pluripotent stem cells: perspectives for transfusion medicine," Haematologica, vol. 95, no. 10, pp. 1651-1659, 2010.

[37] J. Dias et al., "Generation of red blood cells from human induced pluripotent stem cells," Stem Cells and Development. In press.

[38] E. Szabo, S. Rampalli, R. M. Risueño et al., "Direct conversion of human fibroblasts to multilineage blood progenitors," Nature, vol. 468, no. 7323, pp. 521-526, 2010.

[39] R. C. Perlingeiro, M. Kyba, and G. Q. Daley, "Clonal analysis of differentiating embryonic stem cells reveals a hematopoietic progenitor with primitive erythroid and adult lymphoidmyeloid potential," Development, vol. 128, no. 22, pp. $4597-$ 4604, 2001.

[40] K. H. Chang, A. M. Nelson, H. Cao et al., "Definitive-like erythroid cells derived from human embryonic stem cells coexpress high levels of embryonic and fetal globins with little or no adult globin," Blood, vol. 108, no. 5, pp. 1515-1523, 2006.

[41] A. Seifinejad, A. Taei, M. Totonchi et al., "Generation of human induced pluripotent stem cells from a Bombay individual: moving towards "universal-donor" red blood cells," Biochemical and Biophysical Research Communications, vol. 391, no. 1, pp. 329-334, 2010.

[42] J. Hanna, M. Wernig, S. Markoulaki et al., "Treatment of sickle cell anemia mouse model with iPS cells generated from autologous skin," Science, vol. 318, no. 5858, pp. 1920-1923, 2007.

[43] L. C. Wu, C. W. Sun, T. M. Ryan, K. M. Pawlik, J. Ren, and T. M. Townes, "Correction of sickle cell disease by homologous recombination in embryonic stem cells," Blood, vol. 108, no. 4, pp. 1183-1188, 2006. 

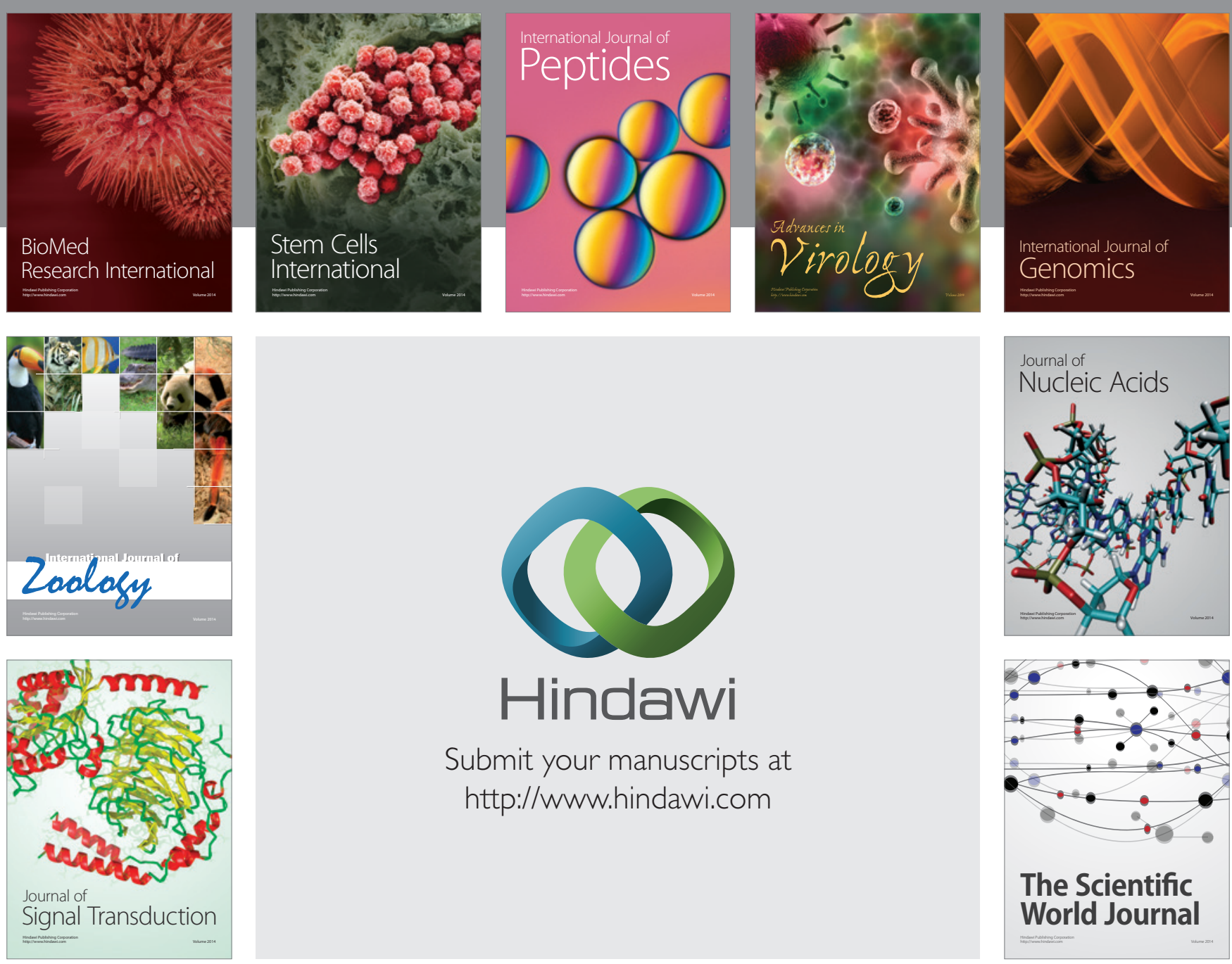

Submit your manuscripts at

http://www.hindawi.com
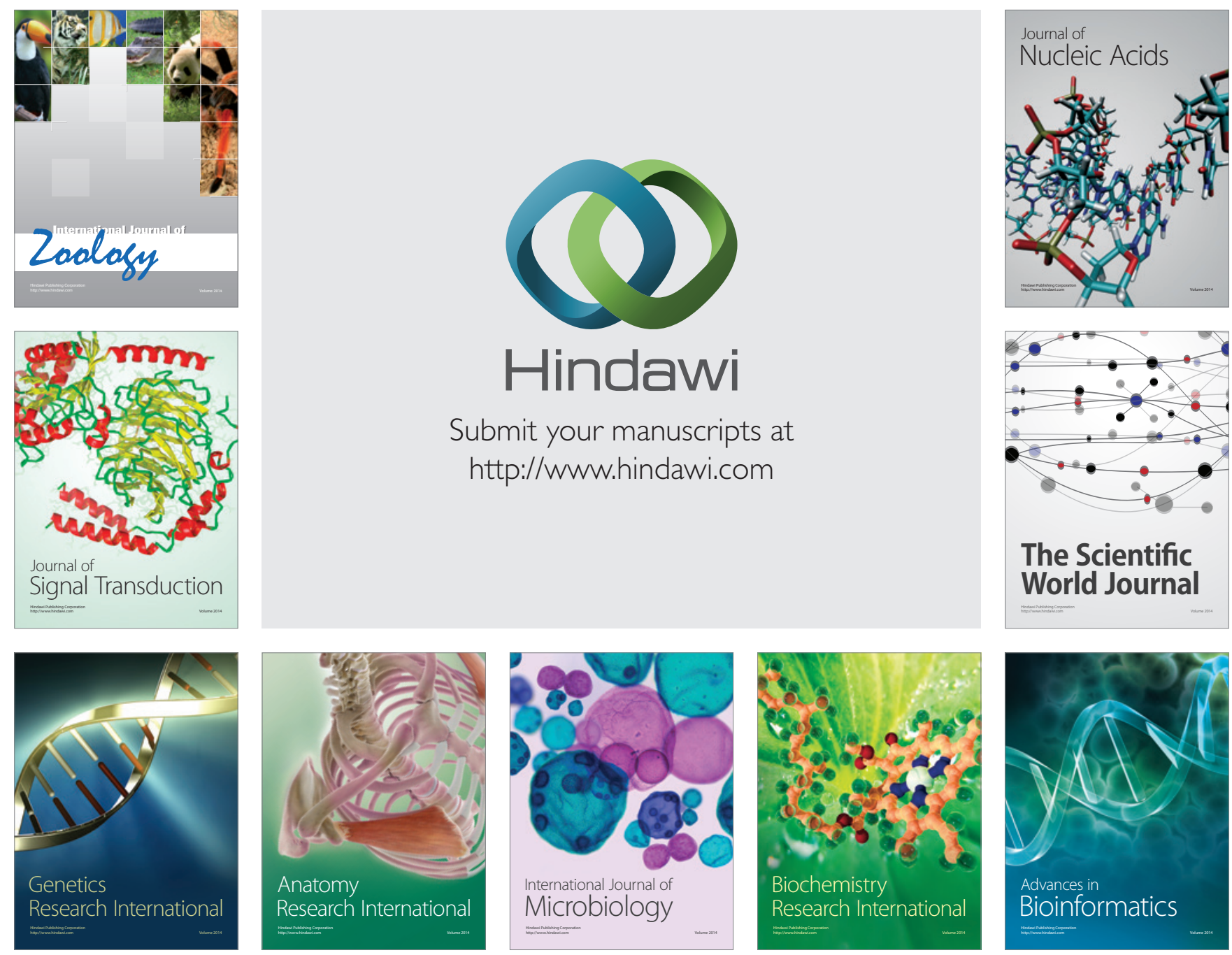

The Scientific World Journal
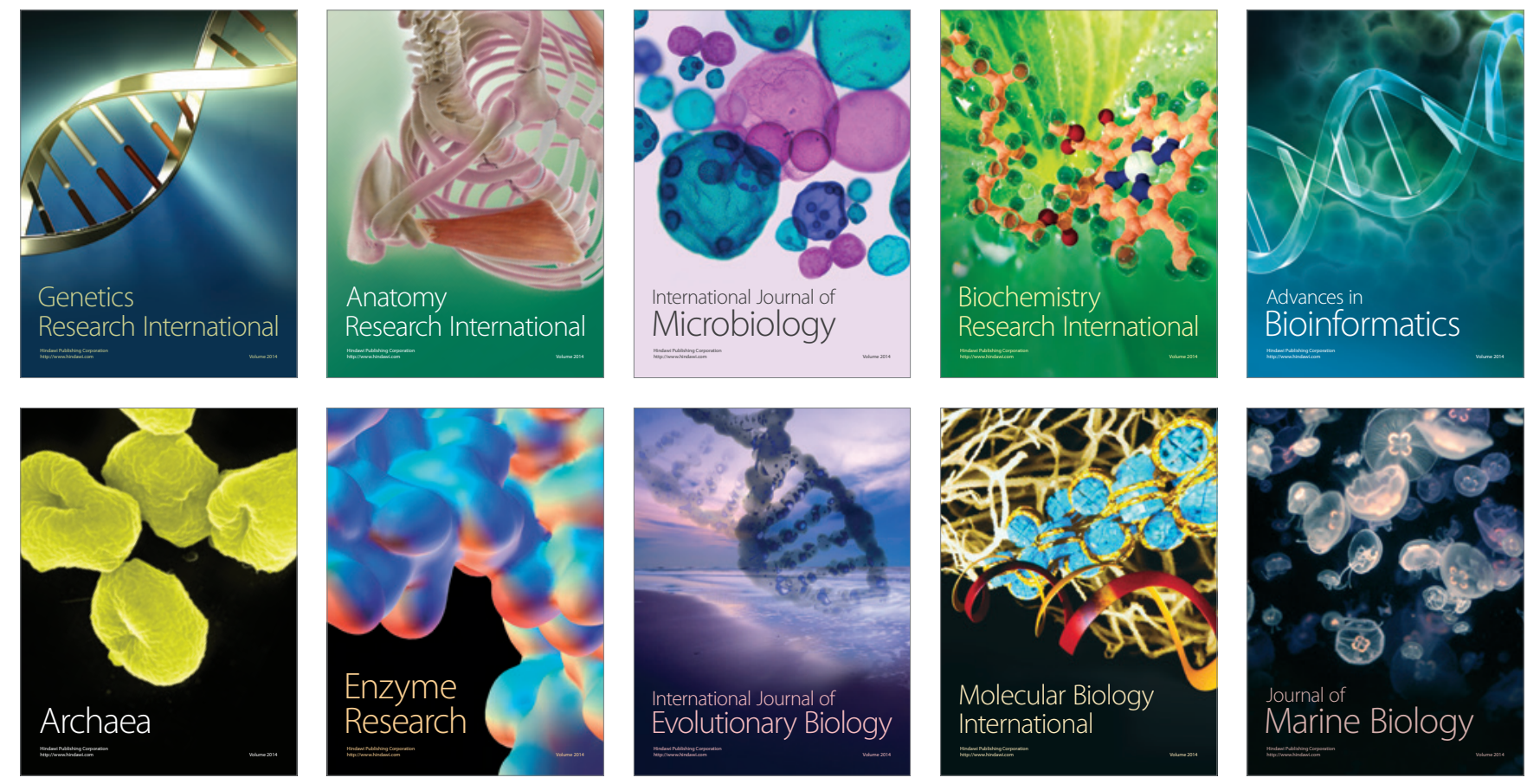